\title{
Boundary state bootstrap and asymptotic overlaps in AdS/dCFT
}

\author{
Tamas Gombor and Zoltan Bajnok \\ Wigner Research Centre for Physics, \\ Konkoly-Thege Miklós u. 29-33, 1121 Budapest, Hungary \\ E-mail: gombor.tamas@wigner.mta.hu, bajnok.zoltan@wigner.mta.hu
}

ABSTRACT: We formulate and close the boundary state bootstrap for factorizing K-matrices in AdS/CFT. We found that there are no boundary degrees of freedom in the boundary bound states, merely the boundary parameters are shifted. We use this family of boundary bound states to describe the D3-D5 system for higher dimensional matrix product states and provide their asymptotic overlap formulas. In doing so we generalize the nesting for overlaps of matrix product states and Bethe states.

Keywords: Integrable Field Theories, AdS-CFT Correspondence, Boundary Quantum Field Theory, Bethe Ansatz

ArXiv EPrint: 2006.16151 


\section{Contents}

1 Introduction 1

2 Boundary state bootstrap $\quad 2$

2.1 Boundary bound state K-matrix 4

2.2 Symmetry considerations 4

2.3 Closing the boundary bootstrap 5

2.3.1 Boundary ground state 6

$\begin{array}{lll}\text { 2.3.2 } & \text { First boundary excited state } & 7\end{array}$

$\begin{array}{lll}2.3 .3 & \text { Second boundary excited state } & 8\end{array}$

2.3.4 Generic boundary excited state $\quad 8$

2.3.5 Closing the bootstrap $\quad 8$

$\begin{array}{lll}2.4 & \text { Weak coupling expansion } & 9\end{array}$

3 Asymptotic AdS/dCFT overlaps 10

$\begin{array}{ll}3.1 \text { Nesting for overlaps with an MPS } & 10\end{array}$

$\begin{array}{lll}3.2 & \mathfrak{s u}(3) \text { spin chain } & 14\end{array}$

$\begin{array}{lll}3.3 & \mathfrak{s o}(6) \text { spin chain } & 16\end{array}$

$\begin{array}{ll}3.4 & \text { Proposal for the asymptotic overlap in AdS/dCFT for generic k }\end{array}$

4 Conclusions $\quad 18$

\section{Introduction}

Boundaries always played significant roles in integrable two dimensional systems [1]. When they are placed in space they provide boundary conditions for the fields, leading to reflection factors for their particle like excitations. When they are placed in time they serve as initial or final states which create or annihilate these particles. Integrable boundary states attracted considerable interest recently both in non-equilibrium statistical physics and the AdS/dCFT correspondence. They showed up in integrable quench problems $[2-5]$ and in the calculation of one point functions in the maximally supersymmetric gauge theory with a codimension one defect [6-16]. In both cases the key object is the overlap of the finite volume boundary state with finite volume multi-particle states. Although most of the applications focused on the overlap of the ground state boundary, recently it was observed in [17] that the description of the higher dimensional matrix product states (MPS) in the D3-D5 system requires to incorporate the boundary bound states, too. In [17] the authors focused on the diagonal $\mathfrak{s u}(2)$ sub-sector of the theory. Our aim is to extend the boundary bootstrap procedure for the whole theory including the full matrix structure. In doing so we develop the bootstrap procedure for boundary states. 
Integrable boundary states can be equivalently described by the K-matrix, which encodes how pairs of particles are annihilated. This K-matrix is in one-to-one correspondence with the one-particle reflection matrix in the mirror theory. The boundary Yang-Baxter equation (BYBE) of the mirror theory is equivalent to the KYBE of the original theory, which is the consistency equation of the K-matrix [18]. Much is known about the boundary bootstrap, when poles of the reflection factor are explained either by boundary bound states or by some boundary Coleman-Thun diagrams [19-23]. If the theory and the mirror theory is not equivalent, as in the AdS/CFT correspondence, then the boundary bootstrap for the reflection factor is not equivalent to the boundary bootstrap for the boundary state. This boundary state bootstrap, which is equivalent to the boundary bootstrap for the mirror reflection factor, can be formulated more intuitively in the original theory. It amounts to explain the poles of the K-matrix in terms of excited boundary states and Coleman-Thun diagrams, diagrams with on-shell propagating particles with energy and momentum preserving point-like interactions [23]. Our aim is to carry out the boundary state bootstrap for the K-matrix appearing in the D3-D5 system of the AdS/dCFT correspondence and extend the result of $[17,18]$. In doing so we need the full excitation K-matrix of the D3-D5 system at tree level. By generalizing the nesting methods of [18] for matrix product states we calculate these K-matrices, which, together with the all loop bound state K-matrices, lead to the complete asymptotic overlaps.

The paper is organized as follows: In section 2 we formulate and close the boundary bootstrap for the factorizing K-matrices we found in [18]. We then generalize the nesting for matrix product states' overlaps and extract the tree level excitation K-matrices by explicit calculations in section 3. In the end of section 3 we propose our formulas for the full asymptotic overlap including all sectors. Finally, we conclude in section 4.

\section{Boundary state bootstrap}

In the $A d S_{5} / C F T_{4}$ correspondence the scattering matrix factorizes into the product of two $\mathfrak{s u}(2 \mid 2)$ invariant scattering matrices [24]:

$$
\mathbb{S}\left(p_{1}, p_{2}\right)=S_{0}\left(p_{1}, p_{2}\right) S\left(p_{1}, p_{2}\right) \otimes S\left(p_{1}, p_{2}\right)
$$

In our previous paper we have determined the most general factorizing solutions

$$
\mathbb{K}(p)=K_{0}(p) K(p) \otimes K(p)
$$

of the KYBE without inner degrees of freedom [18]. We found that they all have centrally extended $\mathfrak{o s p}(2 \mid 2)_{c}$ residual symmetry and the various solutions were characterized by how this symmetry can be embedded into the centrally extended $\mathfrak{s u}(2 \mid 2)_{c}$. There were two classes depending on whether the unbroken $\mathfrak{s u}(2)$ symmetry was in the Lorentz or in the $R$-symmetry part. As these two cases are quite analogous we focused on the solution, which 
preserves the Lorentz symmetry and can be written as

$$
K(p)=\left(\begin{array}{cccc}
k_{1} & k_{2}+e(p) & 0 & 0 \\
k_{2}-e(p) & k_{4} & 0 & 0 \\
0 & 0 & 0 & f(p) \\
0 & 0 & -f(p) & 0
\end{array}\right) ; \quad \begin{aligned}
e(p) & =-i \frac{x_{s}^{2} x^{-}+x^{+}}{x_{s}\left(1+x^{+} x^{-}\right)} \\
k_{1} k_{4}-k_{2}^{2} & =1
\end{aligned}
$$

where $x^{ \pm} \equiv x^{ \pm}(p)$ are the standard parameters of the one-particle representations. This parametrization is slightly different from [18] by rescaling $k_{i}$, which now parameterize the orientation of the bosonic part of the $\mathfrak{o s p}(2 \mid 2)_{c} \subset \mathfrak{s u}(2 \mid 2)_{c}$ embedding, while the parameter ${ }^{1}$ $x_{s}$ is responsible for the fermionic orientation $\tilde{\mathbb{Q}}_{\alpha}^{a}=\mathbb{Q}_{\alpha}{ }^{a}+i x_{s}^{-1} \epsilon_{\alpha \beta} \sigma_{1}^{a b} \mathbb{Q}_{b}^{\dagger \beta}$, with $\sigma_{1}$ being the first Pauli matrix. Our normalization is also different from [18], as we removed the factor $\frac{-i x_{s}\left(1+x^{+} x^{-}\right)}{\left(x^{+}\right)^{2}-x_{s}^{2}}$ and put it directly in to $K_{0}(p)$. This choice is more useful for bootstrap purposes and the boundary crossing equation takes also a very simple form

$$
K_{0}(p)=S_{0}(p,-p) K_{0}(p) ; \quad S_{0}\left(p_{1}, p_{2}\right)=\frac{x_{1}^{-}}{x_{1}^{+}} \frac{x_{2}^{+}}{x_{2}^{-}} \frac{x_{1}^{+}-x_{2}^{-}}{x_{1}^{-}-x_{2}^{+}} \frac{1-\frac{1}{x_{1}^{+} x_{2}^{-}}}{1-\frac{1}{x_{1}^{-} x_{2}^{+}}} \sigma\left(p_{1}, p_{2}\right)^{2}
$$

where $S_{0}\left(p_{1}, p_{2}\right)$ is the scalar factor in the $\mathfrak{s u}(2)$ sector. The $K$-matrix satisfies the KYBE:

$$
K_{23}\left(p_{2}\right) K_{14}\left(p_{1}\right) S_{13}\left(p_{1},-p_{2}\right) S_{13}\left(p_{1}, p_{2}\right)=K_{14}\left(p_{1}\right) K_{23}\left(p_{2}\right) S_{24}\left(p_{2},-p_{1}\right) S_{34}\left(-p_{2},-p_{1}\right)
$$

where the subscripts indicate in which representation spaces the operators act $\left(p_{1}, p_{2},-p_{2},-p_{1}\right)$. The reflection factor of the mirror theory defined by $R(p(z))=$ $C K\left(p\left(\frac{\omega_{2}}{2}-z\right)\right)$ satisfies the boundary Yang-Baxter equation [18]. Here $z$ is the torus rapidity parameter, $C$ is the charge conjugation matrix and $\omega_{2}$ is the crossing parameter. Unitarity of the mirror reflection factor implies the following equation for the scalar factor

$$
K_{0}(p(z+\omega)) K_{0}(p(z))=\frac{x_{s}^{4}\left(1+x^{+} x^{-}\right)^{4}}{\left(\left(x^{+}\right)^{2}-x_{s}^{2}\right)^{2}\left(1-\left(x^{-}\right)^{2} x_{s}^{2}\right)^{2}}
$$

The minimal solution to the crossing (2.4) and unitarity equation (2.6) was found in the D3-D5 setting in [17]. Our conventions are related to those by $x^{ \pm} \leftrightarrow x^{\mp}$ which originate from the different definition of the scattering matrix. ${ }^{2}$ We also have a slightly different normalization of the K-matrix. In this paper we focus on the bootstrap and the matrix part of the solution together with the corresponding overlaps, so we just point out that $K_{0}(z)$ can be obtained from that of [17] by the corresponding changes. In order to keep the discussion in a more general level we assume that the scalar factor has poles at $x^{+}= \pm x_{s}$, which come from the removed factor $\frac{-i x_{s}\left(1+x^{+} x^{-}\right)}{\left(x^{+}\right)^{2}-x_{s}^{2}}$. Following [17] one might regard $x_{s}$ as the $x$ parameter of a boundary rapidity is, which satisfies: $x_{s}+x_{s}^{-1}=\frac{i s}{g}$. The poles signal boundary bound states and in the following we calculate the K-matrices of the corresponding excited boundary state.

\footnotetext{
${ }^{1}$ We introduced $x_{s}$ to conform with the notation of [17]. It is related to $s$ in [18] as $s=-i x_{s}$.

${ }^{2}$ Part of the literature uses the $S$-matrix [25], while some other part the inverse of it [26]. This can be easily pinpointed by how the S-matrix appears in the Bethe ansatz equations. Accordingly, the physical domain of the rapidity is also exchanged in the two conventions. We use the conventions of [26].
} 

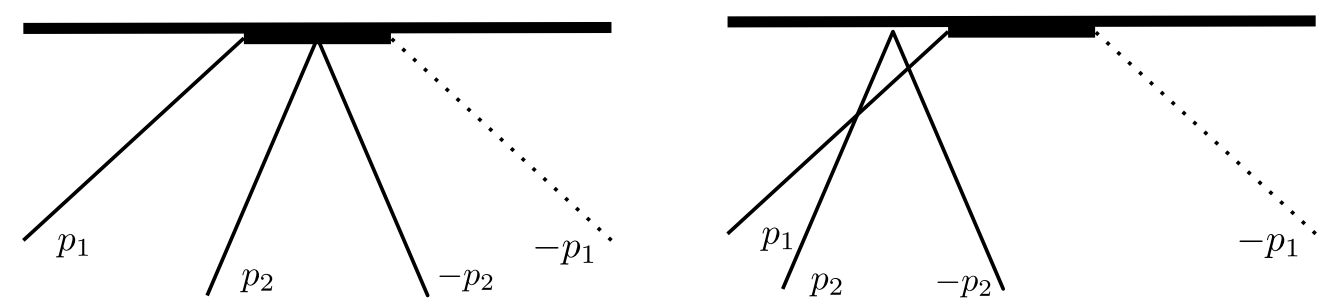

Figure 1. Boundary bootstrap for the K-matrix (boundary state). Trajectories can be shifted allowing to calculate the bound state K-matrix in terms of the groundstate one and scattering matrices.

\subsection{Boundary bound state K-matrix}

The bootstrap principle tells us that the $K$-matrix of the boundary bound state $\bar{K}(p)$ can be calculated from the ground state one by shifting the trajectories of the particles as show on figure 1:

$$
\bar{K}_{23}\left(p_{2}\right) \operatorname{res}_{x_{1}^{+}= \pm x_{s}} K_{14}\left(p_{1}\right)=\operatorname{res}_{x_{1}^{+}= \pm x_{s}}\left(K_{23}\left(p_{2}\right) K_{14}\left(p_{1}\right) S_{13}\left(p_{1},-p_{2}\right) S_{12}\left(p_{1}, p_{2}\right)\right)
$$

It is particularly useful to normalize the $\mathfrak{s u}(2 \mid 2)$ scattering matrix as $S_{11}^{11}\left(p_{1}, p_{2}\right)=1$. By performing the calculation it turns out that the K-matrix at the pole position is proportional to a one dimensional projector thus the bound state K-matrix has the same dimension as the original one. This implies that there is no boundary degree of freedom in the boundary bound state, similarly to the boundary ground state with which we started. Due to the bootstrap construction the bound state K-matrix satisfies the KYBE (2.5) thus has the form (2.3) and can be described by other constants $\bar{s}, \bar{k}_{i}$. On bootstrapping on the poles $x_{1}^{+}= \pm x_{s}$ we found that, due to our special normalization, the parameters of the bound state K-matrix can be obtained from the original one as

$$
s \rightarrow \bar{s}=s \mp 1 ; \quad k_{i} \rightarrow \bar{k}_{i}
$$

and the scalar factor changes as

$$
\bar{K}_{0}(p)=S_{0}\left(p_{s}, p\right) S_{0}\left(p_{s},-p\right) K_{0}(p) ; \quad x^{+}\left(p_{s}\right)= \pm x_{s}
$$

Similar calculations can be done for the other $\mathfrak{o s p}(2 \mid 2)_{c}$ residual symmetry, when the unbroken $\mathfrak{s u}(2)$ part is the R-symmetry with the same conclusion, i.e. there is no boundary degrees of freedom and the parameters change the same way: $s \rightarrow \bar{s}=s \mp 1$ depending on which pole we bootstrap on and the $\bar{k}$ s are not changed.

\subsection{Symmetry considerations}

These results can be easily understood from symmetry considerations. Indeed, the fermionic symmetry of the K-matrix can be written formally as [18]

$$
K(p) \Delta\left(\mathbb{Q}+x_{s}^{-1} \mathbb{Q}^{\dagger}\right)=0
$$


The shift in the parameter $s$ comes from the different co products of $\mathbb{Q}$ and $\mathbb{Q}^{\dagger}$ :

$$
\Delta(\mathbb{Q})=\mathbb{Q} \otimes e^{i \mathbb{P} / 4}+e^{-i \mathbb{P} / 4} \otimes \mathbb{Q}, \quad \Delta\left(\mathbb{Q}^{\dagger}\right)=\mathbb{Q}^{\dagger} \otimes e^{-i \mathbb{P} / 4}+e^{i \mathbb{P} / 4} \otimes \mathbb{Q}^{\dagger}
$$

via the bootstrap procedure, which is obtained through the pole term of the KYBE at $x^{+}\left(p_{1}\right)= \pm x_{s}$. Since the KYBE (2.5) is an equation on the product of one particle $\mathfrak{s u}(2 \mid 2)_{c}$ representations with momenta $\mathcal{V}\left(p_{1}\right) \otimes \mathcal{V}\left(p_{2}\right) \otimes \mathcal{V}\left(-p_{2}\right) \otimes \mathcal{V}\left(-p_{1}\right)$ the co-product of the supercharges takes the following form

$$
\begin{aligned}
\Delta^{(4)}(\mathbb{Q}) & =e^{-i \frac{p_{1}}{4}} \mathbb{Q} \otimes \mathbb{I} \otimes \mathbb{I} \otimes \mathbb{I}+e^{-i \frac{p_{1}}{4}} \mathbb{I} \otimes \mathbb{I} \otimes \mathbb{I} \otimes \mathbb{Q}+e^{-i p_{1} / 2}(\mathbb{I} \otimes \Delta(\mathbb{Q}) \otimes \mathbb{I}) \\
& =e^{-i \frac{p_{1}}{4}}\left(\mathbb{Q}_{1}+\mathbb{Q}_{4}\right)+e^{-i p_{1} / 2} \Delta_{23}(\mathbb{Q}) .
\end{aligned}
$$

This can be decomposed with respect to the particle types as the action of the charge on the representations with momentum $p_{1}$ and $-p_{1}$ (first two terms) and $e^{-i p_{1} / 2}$ times the action of the charge on the representations with momentum $p_{2}$ and $-p_{2}$ (second term). In a similar manner the action of $\mathbb{Q}^{\dagger}$ comes with a factor $e^{i p_{1} / 2}$ when acting in the representations of the second particle:

$$
\Delta^{(4)}\left(\mathbb{Q}^{\dagger}\right)=e^{i \frac{p_{1}}{4}}\left(\mathbb{Q}_{1}^{\dagger}+\mathbb{Q}_{4}^{\dagger}\right)+e^{i p_{1} / 2} \Delta_{23}\left(\mathbb{Q}^{\dagger}\right) .
$$

We now act with $\Delta^{(4)}\left(\mathbb{Q}+x_{s}^{-1} \mathbb{Q}^{\dagger}\right)$ on the bootstrap equation (2.7): the r.h.s. is zero since the scattering matrices commute with the charges and the boundary states $K_{14}$ and $K_{23}$ are annihilated by $\Delta\left(\mathbb{Q}+x_{s}^{-1} \mathbb{Q}^{\dagger}\right)$, which implies:

$$
\left[\bar{K}_{23}\left(p_{2}\right) \operatorname{res}_{x_{1}^{+}= \pm x_{s}} K_{14}\left(p_{1}\right)\right] \Delta^{(4)}\left(\mathbb{Q}+x_{s}^{-1} \mathbb{Q}^{\dagger}\right)=0 .
$$

Using (2.12) and the fact that

$$
K_{14}\left(p_{1}\right)\left(e^{-i \frac{p_{1}}{4}}\left(\mathbb{Q}_{1}+\mathbb{Q}_{4}\right)+x_{s}^{-1} e^{i \frac{p_{1}}{4}}\left(\mathbb{Q}_{1}^{\dagger}+\mathbb{Q}_{4}^{\dagger}\right)\right)=0
$$

we can obtain the following property of the bound state K-matrix:

$$
\bar{K}\left(p_{2}\right) \Delta\left(\mathbb{Q} e^{-i \frac{p_{1}}{2}}+x_{s}^{-1} e^{i \frac{p_{1}}{2}} \mathbb{Q}^{\dagger}\right)=0
$$

This shows that the parameter $x_{s}= \pm x_{1}^{+}$in the bound state K-matrix is shifted compared to the original one as:

$$
\bar{K}(z) \Delta\left(\mathbb{Q}+x_{\bar{s}}^{-1} \mathbb{Q}^{\dagger}\right)=0 ; \quad x_{\bar{s}}=e^{-i p_{1}} x_{s}= \pm x_{1}^{-}=x_{s \mp 1}
$$

\subsection{Closing the boundary bootstrap}

The newly calculated bound state $K$-matrix $\bar{K}$ with parameter $\bar{s}$ has the same structure as the ground state one except that the parameter is changed $s \rightarrow \bar{s}$. This signals an another boundary bound state which we can excite by binding a particle with $x^{+}(p)= \pm x_{\bar{s}}$ to the already excited boundary. The boundary bootstrap formulates how this procedure goes. The closure of the bootstrap requires that we explain all poles of all the excited K-matrices. Explanation means that for each singularity we draw a boundary Coleman-Thun diagram 
with on-shell propagating particles. In relativistic theories this is the Coleman-Norton interpretation of the Landau equations coming from all loop perturbative calculations which explains the pole singularities [23].

In performing a complete analysis of the possible bound states the proper scalar factor is essential. So from now on we focus only on the D3-D5 system, where the scalar factor is known [17]. This scalar factor was fixed by solving the crossing (2.4) and unitarity (2.6) equations for $K_{0}$. In our K-matrix we have a prefactor $\left(x^{+}-x_{s}\right)\left(x^{+}+x_{s}\right)$ in the denominator indicating two possible bound states. However, in the solution for $K_{0}$ it is replaced by the following structure:

$$
\frac{\left(x^{+}+\frac{1}{x^{+}}\right)\left(x^{+}+\frac{1}{x^{+}}+x^{-}+\frac{1}{x^{-}}\right)}{2\left(x^{+}-x_{s}\right)\left(1-\frac{1}{x^{+} x_{s}}\right)\left(x^{-}+x_{s}\right)\left(1+\frac{1}{x^{-} x_{s}}\right)} \times \text { regular }
$$

It is thus advantageous to introduce the rapidity variable also for the particle, similarly to the boundary parameter:

$$
x^{ \pm}(p)+\frac{1}{x^{ \pm}(p)}=\frac{u(p) \pm \frac{i}{2}}{g} ; \quad x_{s}+\frac{1}{x_{s}}=\frac{i s}{g}
$$

such that the scalar factor has the form

$$
K_{0}(u)=\frac{u\left(u+\frac{i}{2}\right)}{\left(u-i\left(s-\frac{1}{2}\right)\right)\left(u+i\left(s-\frac{1}{2}\right)\right)} K_{0}^{\mathrm{reg}}(u)
$$

This result is the conjugate expression $\left(x^{ \pm} \leftrightarrow x^{\mp}\right)$ of the one in [17], coming from the fact that we use a different convention for the scattering matrix [26]. This conjugation, however will not effect the final physical overlaps. The regular part contains the ratio of the boundary dressing phase and the bulk dressing phase and will not be relevant for closing the bootstrap which we perform now. ${ }^{3}$ We start with the boundary ground state. The physical domain of the $x$ parameters is $\left|x^{ \pm}\right|>1$ thus, when we have a pole in $u$ at $i\left(s-\frac{1}{2}\right)$, we bootstrap on the pole $x^{+}=x_{s}$ and not on the pole at $x^{+}=\frac{1}{x_{s}}$. This is also true for all bound states.

\subsubsection{Boundary ground state}

In the K-matrix we have two poles in $K_{0}\left(u_{1}\right)$. One at $u_{1}=i\left(s-\frac{1}{2}\right)$ and another one at $u_{1}=-i\left(s-\frac{1}{2}\right)$. At $u_{1}=i\left(s-\frac{1}{2}\right)$ the K-matrix is a rank one projector and the pole is explained by a boundary bound state without any inner degree of freedom as shown on the left of figure 2 .

Due to the boundary crossing unitarity for any pole in the K-matrix at $u_{p}$ there is also a pole at $-u_{p}$. This is similar how in the bulk bootstrap the crossing invariant scattering matrix has poles both in the $s$ - and also in the $t$ - channel. In order to decide which is the boundary bound state we can analyze the $K$-matrix at the pole position. Clearly in our case at the pole $u_{1}=i\left(s-\frac{1}{2}\right)$ we have a one dimensional projector, so it is natural to

\footnotetext{
${ }^{3}$ The boundary dressing phase does not have any pole in the physical domain, while the singularities of the bulk dressing phase correspond to bulk bound states which we do not analyze here.
} 

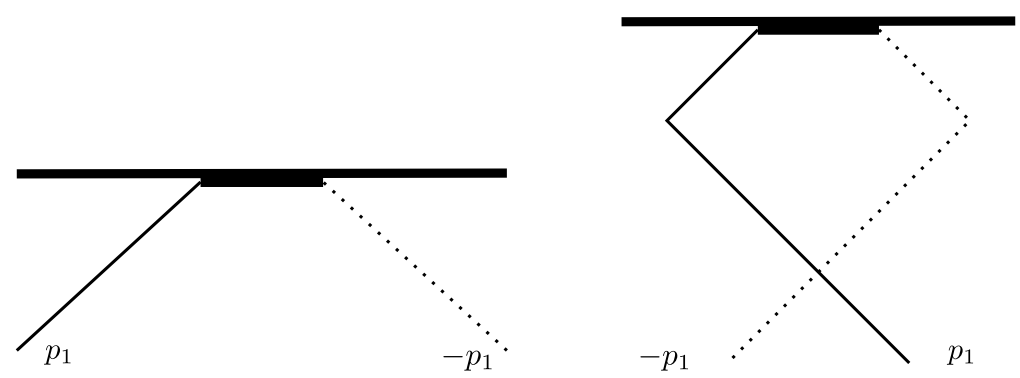

Figure 2. Coleman-Thun diagrams for the poles of the ground state K-matrix. They correspond to a boundary bound state pole in the direct and one in the crossed diagram indicated by a thicker boundary line.

associate a boundary bound state to it. At the position $u_{1}=-i\left(s-\frac{1}{2}\right)$ we have a three dimensional projector as we have poles also in the 34 and 43 matrix elements, but they can be explained by the diagram on the right of figure 2. As 34 can scatter into 12 which creates the bound state at $u_{1}$ we effectively created the bound state by the 34 process at $-u_{1}$. If, instead, we had assumed that the three dimensional projector corresponds to the physical bound state then we would not have been able to use the crossed channel diagram to explain the one dimensional projector structure of the other pole. Thus keeping the first option and using the two diagrams we explained all the ground state poles and proceed with bootstrapping the K-matrix for the boundary bound state.

\subsubsection{First boundary excited state}

We now use the boundary state bootstrap we described in the beginning of the section to calculate the bound state K-matrix and the scalar factor (2.7). This means that a particle with label 1 and momentum $x_{1}^{+}=x_{s}$, binds to the boundary. By bootstrapping on the $u_{1}=i\left(s-\frac{1}{2}\right)$ pole we obtain the excited boundary K-matrix, $\bar{K}\left(u_{2}\right)$ with parameter $\bar{s}=s-1$. The scalar factor can be bootstrapped as

$$
\begin{aligned}
K_{1}\left(u_{2}\right) & =K_{0}\left(u_{2}\right) S_{0}\left(u_{1}, u_{2}\right) S_{0}\left(u_{1},-u_{2}\right) \\
& =\frac{u_{2}\left(u_{2}+\frac{i}{2}\right)}{\left(u_{2}-i\left(s-\frac{1}{2}\right)\right)\left(u_{2}+i\left(s-\frac{1}{2}\right)\right)} \frac{u_{1}-u_{2}+i}{u_{1}-u_{2}-i} \frac{u_{1}+u_{2}+i}{u_{1}+u_{2}-i} K_{1}^{\mathrm{reg}}\left(u_{2}\right)
\end{aligned}
$$

Using that $u_{1}=i\left(s-\frac{1}{2}\right)$ we obtain the excited boundary state scalar factor

$$
K_{1}(u)=\frac{u\left(u+\frac{i}{2}\right)}{\left(u-i\left(s-\frac{1}{2}\right)\right)\left(u+i\left(s-\frac{1}{2}\right)\right)} \frac{u-i\left(s+\frac{1}{2}\right)}{u-i\left(s+\frac{3}{2}\right)} \frac{u+i\left(s+\frac{1}{2}\right)}{u+i\left(s-\frac{3}{2}\right)} K_{1}^{\mathrm{reg}}(u)
$$

Observe that additionally to the pair of ground state poles we obtained a new pair shifted by 1 according to the new $s$ parameter.

The ground state pole always appears in the excited state and can be explained by the diagram on figure 3. There is a crossed version of this diagram, which explains the pole at $-u_{1}$. At these positions the scattering matrix is not a projector. This is not a problem however, since before reaching the boundary the particles have to scatter on each 


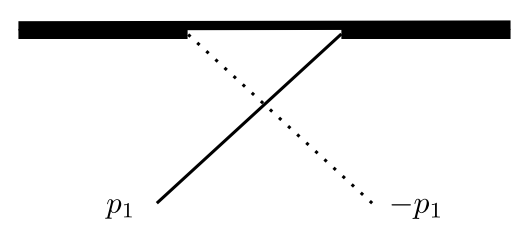

Figure 3. Coleman-Thun diagram for excited boundary states signaling a pole at the ground state excitation position.

other allowing poles in all amplitudes. In particular, the particles 34 can scatter into 12 or 21 , which then can be emitted by the excited boundary and absorbed by the ground state boundary.

\subsubsection{Second boundary excited state}

The pole at $u=i\left(s-\frac{3}{2}\right)$ signals a new bound state. Performing the bootstrap we assume that a particle with rapidity $u_{2}=i\left(s-\frac{3}{2}\right)$ binds to the already excited boundary. The K-matrix of the newly excited boundary $\bar{K}\left(u_{3}\right)$ has a new s-parameter whose rapidity turns out to be $s-2$ and the scalar factor of a particle with rapidity $u_{3}$ is

$$
\begin{aligned}
K_{2}\left(u_{3}\right) & =K_{1}\left(u_{3}\right) S_{0}\left(u_{2}, u_{3}\right) S_{0}\left(u_{2},-u_{3}\right) \\
& \sim K_{1}\left(u_{3}\right) \frac{u_{2}-u_{3}+i}{u_{2}-u_{3}-i} \frac{u_{2}+u_{3}+i}{u_{2}+u_{3}-i}
\end{aligned}
$$

The numerator of the scattering matrix factors cancel the ground state bound state poles and we obtain

$$
K_{2}(u)=\frac{u\left(u+\frac{i}{2}\right)}{\left(u-i\left(s-\frac{3}{2}\right)\right)\left(u+i\left(s-\frac{3}{2}\right)\right)} \frac{u-i\left(s+\frac{1}{2}\right)}{u-i\left(s-\frac{5}{2}\right)} \frac{u+i\left(s+\frac{1}{2}\right)}{u+i\left(s-\frac{5}{2}\right)} K_{2}^{\mathrm{reg}}(u)
$$

Clearly we have the pole corresponding to decaying and exciting and its crossed versions. Fortunately, the pole at $u=i\left(s-\frac{1}{2}\right)$ disappeared, which would have been very difficult to explain. The new pole signals a new boundary bound state. We can repeat this procedure to obtain the generic $n^{\text {th }}$ excited boundary state.

\subsubsection{Generic boundary excited state}

The generic $n^{\text {th }}$ boundary excited state has a boundary rapidity parameter $s-n$ and prefactor

$$
K_{n}(u) \propto \frac{u\left(u+\frac{i}{2}\right)}{\left(u-i\left(s-n+\frac{1}{2}\right)\right)\left(u+i\left(s-n+\frac{1}{2}\right)\right)} \frac{u-i\left(s+\frac{1}{2}\right)}{u-i\left(s-n-\frac{1}{2}\right)} \frac{u+i\left(s+\frac{1}{2}\right)}{u+i\left(s-n-\frac{1}{2}\right)}
$$

Clearly the first pole is a decay-excitation pole, while the second one is signaling a new boundary bound state.

\subsubsection{Closing the bootstrap}

For generic parameter $s$ the bootstrap never stops and we have an infinite family of boundary bound states. 
The bootstrap can close if the factor $\left(u+i\left(s+\frac{1}{2}\right)\right)$ in the numerator cancels the would be bound state pole at $u-i\left(s-N-\frac{1}{2}\right)$. This happens when

$$
s=\frac{N}{2}
$$

where $N$ is an integer. But we have to be careful in this case. Indeed, if for example $N$ is odd then after several steps we obtain the denominator $(u-i) u^{2}(u+i)$ where the new boundary bound state should be at $u=0$. Double pole, however does not correspond to any bound state. Fortunately, $u$ in the prefactor just transforms the double pole into a single one which is appropriate for a new boundary bound state. For even $N$ similar situation appears around the origin at $\left(u-\frac{i}{2}\right)^{2}\left(u+\frac{i}{2}\right)^{2}$ where the should be single pole is at $u=-\frac{i}{2}$. Clearly the prefactor $u+\frac{i}{2}$ helps again and the bootstrap does not stop around the origin in either case.

Thus we can conclude that for $s=\frac{N}{2}$ with $N$ integer the bootstrap is closed and we have $N+1$ boundary bound states with rapidities $\frac{i}{2}(N, N-2, \ldots, 2-N,-N)$. For the comparison with the weak coupling expansion we take $N=2 j$ to transform the spectrum of rapidities into $i(j, j-1, \ldots, 1-j, j)$. By labeling the bound state with its parameter $m=(j, j-1, \ldots, 1-j, j)$ the corresponding scalar factor is

$$
K_{j, m}(u)=\frac{u\left(u+\frac{i}{2}\right)}{\left(u-\frac{(2 m-1) i}{2}\right)\left(u+\frac{(2 m-1) i}{2}\right)} \frac{\left(u-\frac{i(2 j+1)}{2}\right)\left(u+\frac{i(2 j+1)}{2}\right)}{\left(u-\frac{(2 m+1) i}{2}\right)\left(u+\frac{(2 m+1) i}{2}\right)} K_{j, m}^{\mathrm{reg}}(u)
$$

where, in the notations we made explicit the j-dependence, too. In contrast, the matrix part depends only on $m$ and not on $j$. Let us denote this matrix, (2.3) with $x_{s} \rightarrow x_{m}$, by $K^{(m)}(u)$ such that the full K-matrix becomes

$$
\mathbb{K}^{(j, m)}(u)=K_{j, m}(u) K^{(m)}(u) \otimes K^{(m)}(u)
$$

Let us emphasize that this result goes much beyond [17]. In [17] the authors only calculated the prefactor $(2.27)$ and did not consider the matrix part, $K^{(m)}$, nor explained the other, non-boundstate, poles of the reflection factor or considered the closure of the bootstrap.

In order to match our results to the AdS/dCFT calculations we perform a weak coupling expansion.

\subsection{Weak coupling expansion}

Assuming that $k_{i}$ are coupling independent we can make a weak coupling expansion of the bound state K-matrices easily. The matrix part at leading order takes the form

$$
\lim _{g \rightarrow 0} K^{(m)}(u)=\left(\begin{array}{cccc}
k_{1} & k_{2}+\frac{m}{u+\frac{i}{2}} & 0 & 0 \\
k_{2}-\frac{m}{u+\frac{i}{2}} & k_{4} & 0 & 0 \\
0 & 0 & 0 & 0 \\
0 & 0 & 0 & 0
\end{array}\right)
$$

The weak coupling expansion of the prefactor $K_{j, m}(u)$ is $(2.27)$ without the regular part. This will be suitable to compare to direct AdS/dCFT calculations and decide about the parameter $m$ and $k_{i}$. 


\section{Asymptotic AdS/dCFT overlaps}

In the AdS/dCFT setting a codimension one defect at $x_{4}=0$ is introduced in the $\mathcal{N}=4$ SYM theory, which splits the space into two halves having $\mathrm{SU}(N)$ and $\mathrm{SU}(N+k)$ gauge groups. On the $\mathrm{SU}(N+k)$ side the symmetry is broken into $\mathrm{SU}(N)$ by the nontrivial expectation values of three of the six scalar fields:

$$
\phi_{i}(x)=-\frac{1}{x_{4}}\left(\begin{array}{cc}
\left(S_{i}\right)_{k \times k} & 0_{k \times N} \\
0_{N \times k} & 0_{N \times N}
\end{array}\right) ; \quad i=1,2,3
$$

where $S_{i}$ form a $k$-dimensional representation of the $\mathfrak{s u}(2)$ algebra. The one-point functions of local single trace gauge-invariant operators take the form

$$
\left\langle\mathcal{O}_{\Delta}(x)\right\rangle=\frac{c_{\Delta}}{x_{4}^{\Delta}}
$$

where $\Delta$ is the scaling dimension of the operator. Scaling operators correspond to Bethe states and at tree level the vacuum expectation values can be calculated as overlaps with a matrix product sate

$$
c_{\Delta}=\frac{\left\langle\mathrm{MPS}_{k} \mid \mathbf{u}\right\rangle}{\sqrt{\langle\mathbf{u} \mid \mathbf{u}\rangle}} ; \quad\left\langle\mathrm{MPS}_{k}\right|=\sum_{i_{1}, \ldots, i_{L}} \operatorname{Tr}\left(S_{i_{1}} \ldots S_{i_{L}}\right)\left\langle\phi_{i_{1}}\right| \otimes \cdots \otimes\left\langle\phi_{i_{L}}\right|
$$

In [18] we developed a nesting method to calculate the K-matrices of the excitations in nested Bethe Ansatz systems and analyzed the cases when the matrix product states were in the two dimensional representations of $\mathfrak{s u}(2)$. Here we extend those calculations for generic $k$-dimensional representations. Thus we take generators $S_{1}, S_{2}, S_{3}$ of the $\mathfrak{s u}(2)$ algebra

$$
\left[S_{3}, S_{ \pm}\right]= \pm S_{ \pm}, \quad\left[S_{+}, S_{-}\right]=S_{3} ; \quad S_{ \pm}=\frac{1}{\sqrt{2}}\left(S_{1} \pm i S_{2}\right)
$$

in the $k=2 j+1$ dimensional representation where $S_{3}$ acts diagonally:

$$
S_{3}=\operatorname{diag}(j, j-1, \ldots,-j+1,-j)
$$

and analyze matrix product boundary states with these bond representations. As a warm up we analyze the $\mathfrak{s u}(3)$ spin chain then we turn to the $\mathfrak{s o}(6)$ chain relevant for the D3-D5 brane setup in the AdS/dCFT correspondence. Before doing so we summarize our strategy for the calculations by putting special emphasis on the novel features.

\subsection{Nesting for overlaps with an MPS}

In [18] we developed a nesting method to calculate the overlaps of a two-site boundary state with periodic Bethe states of spin chains. Here we generalize this method for the case when the boundary state is a matrix product state.

Let us summarize the nesting for two-site boundary states

$$
\langle\Psi|=\psi \otimes \cdots \otimes \psi=\psi^{\otimes \frac{L}{2}}
$$


which satisfy the integrability condition

$$
\langle\Psi| t(u)=\langle\Psi| \Pi t(u) \Pi
$$

where $t(u)$ and $\Pi t(u) \Pi$ are the transfer matrix and its space reflected version (see [27] for the definition of integrability). For all of the so far investigated integrable boundary states the normalized overlap with Bethe states have the following factorized form

$$
\frac{\left\langle\Psi \mid \mathbf{u}^{(a)}\right\rangle}{\sqrt{\left\langle\mathbf{u}^{(a)} \mid \mathbf{u}^{(a)}\right\rangle}}=\prod_{a} \prod_{i=1}^{N_{a} / 2} h^{(a)}\left(u_{i}^{(a)}\right) \sqrt{\frac{\operatorname{det} G_{+}}{\operatorname{det} G_{-}}}
$$

where $\Psi$ has a normalized overlap with the pseudovacuum $\langle\Psi \mid 0\rangle=1$ and $G_{ \pm}$are the Gaudin-like determinants [28-30]. These factorized formulas were verified numerically for various systems and integrable states $[3,4,7,8,11,13,14,18,30,32]$ but exact derivations are available only for rank one cases [28, 29, 31]. We emphasize that (3.8) is an exact overlap formula i.e. it holds for any length $L$ and magnon numbers $N_{a}$. The Gaudin-like determinants depend only on the Bethe state and are well known, but the boundary state dependent functions $h^{(a)}(u)$ are not known for general states. The method of [18] does not try to derive the factorized formula (3.8) but, instead, gives an efficient method to determine the unknown functions $h^{(a)}(u)$ assuming that the exact overlap formula has the above factorized form.

Our method determines the magnonic overlap functions $h^{(a)}$ recursively using the nested Bethe ansatz. At each level of the nesting we test the boundary state with a two particle state and calculate the overlap in the large volume $(L)$ limit. Let us see how it goes. In the first step of the nesting, the Bethe vectors are constructed over the pseudovacuum as

$$
\left|\mathbf{u}^{(a)}\right\rangle=\sum_{\mathbf{a}^{(1)}}\left|\mathbf{u}^{(1)}\right\rangle_{\mathbf{a}^{(1)}} \mathcal{F}_{\mathbf{a}^{(1)}}\left(\mathbf{u}^{(a)}\right)
$$

where the vector $\mathbf{a}^{(1)}=\left\{a_{1}^{(1)}, \ldots, a_{N_{1}}^{(1)}\right\}$ labels the first level excitations and $\mathcal{F}_{\mathbf{a}^{(1)}}\left(\mathbf{u}^{(a)}\right)$ is an eigenvector of the second level transfer matrix. At leading order in $L$ these first level excitations are orthogonal and are normalized as

$$
\lim _{L \rightarrow \infty} \mathbf{b}^{(1)}\left\langle\mathbf{u}^{(1)} \mid \mathbf{u}^{(1)}\right\rangle_{\mathbf{a}^{(1)}}=N_{\mathbf{u}^{(1)}}^{2} \delta_{\mathbf{a}^{(1)}, \mathbf{b}^{(1)}}+\ldots
$$

where the dots represent subleading terms in the number of sites, $L$. The norm of the Bethe vectors in this limit is factorized as

$$
\lim _{L \rightarrow \infty}\left\langle\mathbf{u}^{(a)} \mid \mathbf{u}^{(a)}\right\rangle=N_{\mathbf{u}^{(1)}}^{2} \sum_{\mathbf{a}^{(1)}} \mathcal{F}_{\mathbf{a}^{(1)}}\left(\mathbf{u}^{(a)}\right) \mathcal{F}_{\mathbf{a}^{(1)}}\left(\mathbf{u}^{(a)}\right)+\cdots=: N_{\mathbf{u}^{(1)}}^{2}\left\langle\mathbf{u}^{(a)} \mid \mathbf{u}^{(a)}\right\rangle^{(2)},
$$

where we introduced the braket notation for the second level Bethe vectors $\left[\left|\mathbf{u}^{(a)}\right\rangle^{(2)}\right]_{\mathbf{a}^{(1)}}=$ $\mathcal{F}_{\mathbf{a}^{(1)}}\left(\mathbf{u}^{(a)}\right)$. In calculating the normalized overlap

$$
\lim _{L \rightarrow \infty} \frac{\left\langle\Psi \mid \mathbf{u}^{(a)}\right\rangle}{\sqrt{\left\langle\mathbf{u}^{(a)} \mid \mathbf{u}^{(a)}\right\rangle}}=\frac{\sum_{\mathbf{a}^{(1)}}\left\langle\Psi \mid \mathbf{u}^{(1)}\right\rangle_{\mathbf{a}^{(1)}} \mathcal{F}_{\mathbf{a}^{(1)}}\left(\mathbf{u}^{(a)}\right)}{N_{\mathbf{u}^{(1)}} \sqrt{\sum_{\mathbf{a}^{(1)}} \mathcal{F}^{*} \mathbf{a}^{(1)}\left(\mathbf{u}^{(a)}\right) \mathcal{F}_{\mathbf{a}^{(1)}}\left(\mathbf{u}^{(a)}\right)}}=: \frac{\left\langle\Psi\left(\mathbf{u}^{(1)}\right) \mid \mathbf{u}^{(a)}\right\rangle^{(2)}}{\sqrt{\left\langle\mathbf{u}^{(a)} \mid \mathbf{u}^{(a)}\right\rangle^{(2)}}},
$$


we can also introduce the bra notation for the second level boundary state $\left[{ }^{(2)}\left\langle\Psi\left(\mathbf{u}^{(1)}\right)\right|\right]_{\mathbf{a}^{(1)}}=N_{\mathbf{u}^{(1)}}^{-1}\left\langle\Psi \mid \mathbf{u}^{(1)}\right\rangle_{\mathbf{a}^{(1)}}$. By choosing the normalization of the two-site state as $\langle\Psi \mid 0\rangle=1$ we have ensured a finite $L \rightarrow \infty$ limit. Integrability of the second level boundary state, however, requires an asymptotic factorization of the form:

$$
\lim _{L \rightarrow \infty}{ }^{(2)}\left\langle\Psi\left(\mathbf{u}^{(1)}\right)\right|=K^{(1)}\left(u_{1}\right) \otimes \cdots \otimes K^{(1)}\left(u_{N_{1} / 2}\right) .
$$

It is not clear how this follows from the integrability of the state $\Psi$ and is an assumption in our approach. We validated this assumption in [18] by calculating already known overlaps with this method and we saw that the results were consistent. By choosing the label of the second level pseudo vacuum to be 1 , the overlap with the second level pseudovacuum $|0\rangle^{(2)}$ is simply

$$
\frac{\left\langle\Psi\left(\mathbf{u}^{(1)}\right) \mid 0\right\rangle^{(2)}}{\sqrt{\langle 0 \mid 0\rangle^{(2)}}}=\prod_{i=1}^{N_{1} / 2} K_{11}^{(1)}\left(u_{i}^{(1)}\right)
$$

It is natural to identify the result with the level one overlap function:

$$
h^{(1)}(u):=K_{11}^{(1)}(u)
$$

Since in the known cases the overlap functions $h^{(a)}$ does not depend on the inhomogeneities we define the second level two site state as

$$
{ }^{(2)}\left\langle\Psi\left|=\psi^{(2)} \otimes \cdots \otimes \psi^{(2)} ; \quad \psi^{(2)}:=\frac{K^{(1)}(u)}{h^{(1)}(u)}\right|_{u=0}\right.
$$

This second level two-site state is normalized as $\langle\Psi \mid 0\rangle^{(2)}=1$ and can be used to repeat the nesting procedure at the second level. Repeating this procedure we can calculate all overlap functions $h^{(a)}(u)$, recursively. We emphasize that this method uses assumptions like (3.13) at all levels of nesting.

Let us finally note that if $\Psi$ were not normalized i.e. the overlap of the two site state with the pseudo vacuum were $\psi_{11}=A^{2}$, implying $\langle\Psi \mid 0\rangle=A^{L} \neq 1$, then the asymptotic limit of the second level final state would have this extra factor:

$$
\lim _{L \rightarrow \infty}{ }^{(2)}\left\langle\Psi\left(\mathbf{u}^{(1)}\right)\right|=A^{L} K^{(1)}\left(u_{1}\right) \otimes \cdots \otimes K^{(1)}\left(u_{N_{1} / 2}\right)
$$

This factor also shows up in the full overlap formula

$$
\frac{\left\langle\Psi \mid \mathbf{u}^{(a)}\right\rangle}{\sqrt{\left\langle\mathbf{u}^{(a)} \mid \mathbf{u}^{(a)}\right\rangle}}=A^{L} \prod_{a} \prod_{i=1}^{N_{a} / 2} h^{(a)}\left(u_{i}^{(a)}\right) \sqrt{\frac{\operatorname{det} G_{+}}{\operatorname{det} G_{-}}} .
$$

Let us generalize this method to MPS:

$$
\langle\mathrm{MPS}|=\sum_{i_{1} \ldots i_{L}} \operatorname{Tr}\left[\omega_{i_{1}} \ldots \omega_{i_{L}}\right]\left\langle i_{1} \ldots i_{L}\right|
$$


where $\omega_{k} \in \operatorname{End}\left(V_{B}\right), V_{B}$ is the boundary vector space. In the calculation above, we did not use the periodic boundary condition, therefore in the generalization we cut the trace and define the matrix valued MPS in the boundary space as:

$$
{ }_{\alpha \beta}\langle\mathrm{MPS}|=\sum_{i_{1} \ldots i_{L}}\left(\omega_{i_{1}} \ldots \omega_{i_{L}}\right)_{\alpha \beta}\left\langle i_{1} \ldots i_{L}\right| .
$$

In calculating the normalized overlap

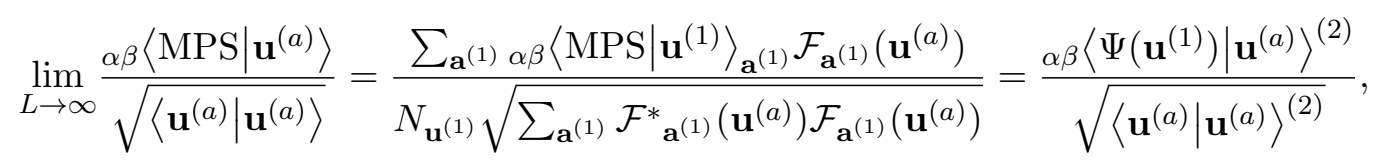

we also introduce the bra notation for the second level final state

$$
\left[{ }_{\alpha \beta}^{(2)}\left\langle\Psi\left(\mathbf{u}^{(1)}\right)\right|\right]_{\mathbf{a}^{(1)}}=\frac{\alpha \beta\left\langle\mathrm{MPS} \mid \mathbf{u}^{(1)}\right\rangle_{\mathbf{a}^{(1)}}}{N_{\mathbf{u}^{(1)}}} .
$$

We have to calculate this overlap for all $\alpha, \beta$. It is obvious, however, that all components can not be normalized as $\alpha \beta\langle\mathrm{MPS} \mid 0\rangle=1$ simultaneously. This implies $A^{L}$-like factors even at the asymptotic limit, which has to be taken carefully.

Usually, the asymptotic second level final state is not irreducible in the boundary space, rather it can be decomposed into irreducible components as

$$
\lim _{L \rightarrow \infty}{ }_{\alpha \beta}^{(2)}\left\langle\Psi\left(\mathbf{u}^{(1)}\right)\right|=\left(\left\langle\Psi_{1}\left(\mathbf{u}^{(1)}\right)\right| \oplus_{V_{B}}\left\langle\Psi_{2}\left(\mathbf{u}^{(1)}\right)\right| \cdots \oplus_{V_{B}}\left\langle\Psi_{n}\left(\mathbf{u}^{(1)}\right)\right|\right)_{\alpha \beta}
$$

where $\operatorname{dim}_{V_{B}}\left(\left\langle\Psi_{i}\left(\mathbf{u}^{(1)}\right)\right|\right)=d_{i}$. We assume that the matrix part is factorized into a product of two-particle (matrix valued) K-matrices in the asymptotic limit, i.e., $\left\langle\Psi_{m}\left(\mathbf{u}^{(1)}\right)\right|$ can be written as

$$
{ }_{\alpha \beta}\left\langle\Psi_{m}\left(\mathbf{u}^{(1)}\right)\right|=A_{m}^{L} K_{\alpha \gamma_{1}}^{(1, m)}\left(u_{1}^{(1)}\right) \otimes K_{\gamma_{1} \gamma_{2}}^{(1, m)}\left(u_{2}^{(1)}\right) \otimes \cdots \otimes K_{\gamma_{N_{1} / 2} \beta}^{(1, m)}\left(u_{N_{1} / 2}^{(1)}\right)
$$

where the excitation K-matrices $K_{\alpha \beta}^{(1, m)}(u)$ do not depend on $L$. Applying this formula for the pseudovacuum $\left(\mathbf{u}^{(1)}=\{\}\right)$ gives:

$$
{ }_{\alpha \beta}\left\langle\Psi_{m}(\{\})\right|=A_{m}^{L} \delta_{\alpha \beta}
$$

therefore

$$
{ }_{\alpha \beta}\langle\operatorname{MPS} \mid 0\rangle=\left(A_{1}^{L} \mathbf{1}_{d_{1}} \oplus_{V_{B}} A_{2}^{L} \mathbf{1}_{d_{2}} \oplus_{V_{B}} \cdots \oplus_{V_{B}} A_{n}^{L} \mathbf{1}_{d_{n}}\right)_{\alpha \beta} .
$$

This equation implies that we can obtain $A_{k}$ s from the vacuum overlap and it suggests the irreducible decomposition.

In the following we focus on the case when the irreducible second level states are one dimensional:

$$
\left\langle\Psi_{m}\left(\mathbf{u}^{(1)}\right)\right|=A_{m}^{L} K^{(1, m)}\left(u_{1}^{(1)}\right) \otimes K^{(1, m)}\left(u_{2}^{(1)}\right) \otimes \cdots \otimes K^{(1, m)}\left(u_{N_{1} / 2}^{(1)}\right),
$$


such that we can apply the previous method for each one dimensional part. This amounts to define first level one particle functions and second level two-site states as

$$
h_{m}^{(1)}(u):=K_{11}^{(1, m)}(u) ; \quad \psi_{m}^{(2)}:=\left.\frac{K^{(1, m)}(u)}{h_{m}^{(1)}(u)}\right|_{u=0}
$$

We can now iteratively calculate the one particle overlaps for each irreducible piece and the proposed final overlap takes the form

$$
\frac{\left\langle\operatorname{MPS} \mid \mathbf{u}^{(a)}\right\rangle}{\sqrt{\left\langle\mathbf{u}^{(a)} \mid \mathbf{u}^{(a)}\right\rangle}}=\sum_{m} A_{m}^{L} \prod_{a} \prod_{i=1}^{N_{a} / 2} h_{m}^{(a)}\left(u_{i}^{(a)}\right) \sqrt{\frac{\operatorname{det} G_{+}}{\operatorname{det} G_{-}}} .
$$

For the justification of the used assumptions we carry out this procedure for certain MPS in the $\mathfrak{s u}(3)$ and $\mathfrak{s o}(6)$ spin chains for which the overlaps are already known.

\section{$3.2 \quad \mathfrak{s u}(3)$ spin chain}

Let us consider the following matrix product state

$$
\left\langle\mathrm{MPS}_{k}\right|=\operatorname{Tr}\left[\left(S_{1}\langle 1|+S_{2}\langle 2|+S_{3}\langle 3|\right)^{\otimes L}\right]
$$

and calculate its overlap in the $\mathfrak{s u}(3)$ spin chain with the eigenvectors of the periodic transfer matrix. The excitation K-matrix can be extracted from the large $L$ limit of the overlap between the MPS and a generic two particle state built over the pseudo vacuum which we choose to be $|0\rangle=|3\rangle^{\otimes L}$.

At first let us calculate overlap of the pseudovacuum:

$$
{ }_{\alpha \beta}\left\langle\operatorname{MPS}_{k} \mid 0\right\rangle=\operatorname{diag}\left(j^{L},(j-1)^{L}, \ldots,(-j+1)^{L},(-j)^{L}\right)_{\alpha \beta}
$$

This vacuum overlap suggest that the second level final state is a direct sum of one dimensional states. In coordinate space Bethe ansatz the two particle plane wave takes the form

$$
|u,-u\rangle_{a b}=\sum_{n_{1}=1}^{L} \sum_{n_{2}=n_{1}+1}^{L}\left(e^{i p\left(n_{1}-n_{2}\right)}\left|n_{1} n_{2}\right\rangle_{a b}+e^{-i p\left(n_{1}-n_{2}\right)} S_{a b}^{c d}(2 u)\left|n_{1} n_{2}\right\rangle_{c d}\right) .
$$

where $|n m\rangle_{a b}$ denotes a state with excitations $a=1,2$ at position $n$ and $b=1,2$ at $m$ and their $\mathfrak{s u}(2)$ invariant S-matrix is normalized as $S_{11}^{11}(u)=1$ and so $S_{12}^{21}(u)=\frac{u+i}{u-i}$. The overlap in the large $L$ limit can be written as

$$
\lim _{L \rightarrow \infty} \frac{\alpha \beta\left\langle\operatorname{MPS}_{k} \mid u,-u\right\rangle_{a b}}{N_{u,-u}}=\left(\Sigma_{a b}(u)+S_{a b}^{c d}(2 u) \Sigma_{c d}(-u)\right)_{\alpha \beta}
$$

where

$$
\Sigma_{a b}(u)=\lim _{L \rightarrow \infty} \frac{1}{L} \sum_{n_{1}=1}^{L} \sum_{n_{2}=n_{1}+1}^{L} e^{i p\left(n_{1}-n_{2}\right)} S_{3}^{n_{1}-1} S_{a} S_{3}^{n_{2}-n_{1}-1} S_{b} S_{3}^{L-n_{2}} .
$$


By changing the basis from $a, b=1,2$ to $A, B=+,-$ and using the commutation relations we can calculate

$$
\Sigma_{A B}(u)=\lim _{L \rightarrow \infty} \frac{1}{L} \sum_{n_{1}<n_{2}} e^{i p\left(n_{1}-n_{2}\right)} S_{A} S_{B}\left(S_{3}+A+B\right)^{n_{1}-1}\left(S_{3}+B\right)^{n_{2}-n_{1}-1} S_{3}^{L-n_{2}}
$$

We can then see that $\Sigma_{ \pm \mp}(u)=\operatorname{diag}\left(\Sigma_{ \pm \mp}^{j, j}(u), \Sigma_{ \pm \mp}^{j, j-1}(u), \ldots, \Sigma_{ \pm \mp}^{j,-j+1}(u), \Sigma_{ \pm \mp}^{j,-j}(u)\right)$ are diagonal matrices with entries

$$
\Sigma_{ \pm \mp}^{j, m}(u)=\frac{1}{2}(j(j+1)-m(m \mp 1)) \frac{1}{m\left(e^{i p}-1\right) \pm 1}
$$

while the non-diagonal $\Sigma_{++}(u)$ and $\Sigma_{--}(u)$ components do not have any $O(L)$ piece, thus they vanish in the $L \rightarrow \infty$ limit. In calculating the matrix element $\Sigma_{ \pm \mp}^{j, m}$ we have to normalize the overlap with the pseudo vacuum to 1 . This amounts to renormalizing the overlaps with the factor $m^{L}$ and introduces different normalization for the various diagonal components of the overlap

$$
\begin{aligned}
& \lim _{L \rightarrow \infty} \frac{\alpha \beta\left\langle\mathrm{MPS}_{k} \mid u,-u\right\rangle_{a b}}{N_{u,-u}}= \\
& \operatorname{diag}\left(j^{L} K_{a b}^{(j, j)}(u),(j-1)^{L} K_{a b}^{(j, j-1)}(u), \ldots,(-j+1)^{L} K_{a b}^{(j,-j+1)}(u),(-j)^{L} K_{a b}^{(j,-j)}(u)\right)_{\alpha \beta}
\end{aligned}
$$

where the diagonal scalar K-matrices of the excitations take the form

$$
K_{a b}^{(j, m)}(u)=\frac{u(u+i / 2)\left(u^{2}+(j+1 / 2)^{2}\right)}{\left(u^{2}+(m+1 / 2)^{2}\right)\left(u^{2}+(m-1 / 2)^{2}\right)}\left(\begin{array}{cc}
1 & -\frac{m}{u+i / 2} \\
\frac{m}{u+i / 2} & 1
\end{array}\right)
$$

which means that the first level one-particle overlaps are

$$
h_{m}^{(1)}(u)=\frac{u(u+i / 2)\left(u^{2}+(j+1 / 2)^{2}\right)}{\left(u^{2}+(m+1 / 2)^{2}\right)\left(u^{2}+(m-1 / 2)^{2}\right)}
$$

and the irreducible second level $K$-matrices are

$$
\left(\begin{array}{cc}
1 & -\frac{m}{u+i / 2} \\
\frac{m}{u+i / 2} & 1
\end{array}\right)
$$

Now we can use the known $\mathfrak{s u}(2)$ boundary state overlaps of these $K$-matrices [18]

$$
h_{m}^{(2)}(u)=\frac{\left(u^{2}+m^{2}\right)}{u(u+i / 2)}
$$


to make a proposition for the full overlap. In doing so we have to restore the relative normalization of the various diagonal contributions

$$
\begin{aligned}
\frac{\left\langle\operatorname{MPS}_{k} \mid \mathbf{u}, \mathbf{v}\right\rangle}{\sqrt{\langle\mathbf{u}, \mathbf{v} \mid \mathbf{u}, \mathbf{v}\rangle}} & =\sum_{m=-j}^{j} m^{L} \prod_{i=1}^{N} h_{m}^{(1)}\left(u_{i}\right) \prod_{i=1}^{M} h_{m}^{(2)}\left(v_{i}\right) \sqrt{\frac{\operatorname{det} G_{+}}{\operatorname{det} G_{-}}} \\
& =\sum_{m=-j}^{j} m^{L} \prod_{i=1}^{N} \frac{u_{i}\left(u_{i}+i / 2\right)\left(u_{i}^{2}+(j+1 / 2)^{2}\right)}{\left(u_{i}^{2}+(m+1 / 2)^{2}\right)\left(u_{i}^{2}+(m-1 / 2)^{2}\right)} \prod_{i=1}^{M} \frac{\left(v_{i}^{2}+m^{2}\right)}{v_{i}\left(v_{i}+i / 2\right)} \sqrt{\frac{\operatorname{det} G_{+}}{\operatorname{det} G_{-}}} \\
& =\sum_{m=-j}^{j} m^{L} \frac{Q_{1}(i j+i / 2) Q_{2}(i m)}{Q_{1}(i m+i / 2) Q_{1}(i m-i / 2)} \sqrt{\frac{Q_{1}(0) Q_{1}(i / 2)}{Q_{2}(0) Q_{2}(i / 2)} \frac{\operatorname{det} G_{+}}{\operatorname{det} G_{-}}}
\end{aligned}
$$

where we used the $Q$-functions

$$
Q_{1}(u)=\prod_{i=1}^{N}\left(u-u_{i}\right), \quad Q_{2}(u)=\prod_{i=1}^{N}\left(u-v_{i}\right) .
$$

We can see that (3.42) agrees with the numerically proved formula in $[11,13]$, which verifies that the excitation K-matrix (3.38) we just calculated is physically meaningful.

\section{$3.3 \quad \mathfrak{s o}(6)$ spin chain}

Let us consider the following MPS

$$
\begin{aligned}
{ }_{\alpha, \beta}\left\langle\mathrm{MPS}_{k}\right| & =\left[\left(\sqrt{2} \phi_{1} S_{1}+\sqrt{2} \phi_{3} S_{2}+\sqrt{2} \phi_{5} S_{3}\right)^{\otimes L}\right]^{\alpha, \beta} \\
& =\left[\left(\sqrt{2} \phi_{1} S_{1}+\sqrt{2} \phi_{3} S_{2}+(Z+\bar{Z}) S_{3}\right)^{\otimes L}\right]^{\alpha, \beta}
\end{aligned}
$$

Following the conventions and calculations in [18] we choose the pseudo vacuum as $Z^{\otimes L}$ and label $\mathfrak{s o}(4)$ excitations with $a, b=1, \ldots, 4$. The overlap calculation is similar to the $\mathfrak{s u}(3)$ case the only differences are in the building block

$$
\Sigma_{a b}^{j, m}(u)=\frac{1}{2}\left(\begin{array}{cccc}
\Sigma_{+-}^{j, m}+\Sigma_{-+}^{j, m} & 0 & i \Sigma_{+-}^{j, m}-i \Sigma_{-+}^{j, m} & 0 \\
0 & 0 & 0 & 0 \\
-i \Sigma_{+-}^{j, m}+i \Sigma_{-+}^{j, m} & 0 & \Sigma_{+-}^{j, m}+\Sigma_{-+}^{j, m} & 0 \\
0 & 0 & 0 & 0
\end{array}\right)_{a b}
$$

from which the diagonal $K$-matrix $\left(K_{a b}^{(j, j)}(u), K_{a b}^{(j, j-1)}(u), \ldots, K_{a b}^{(j,-j+1)}(u), K_{a b}^{(j,-j)}(u)\right)$ can be built up as

$$
\begin{aligned}
K_{a b}^{(j, m)}(u) & =\Sigma_{a b}^{j, m}(u)+S_{a b}^{c d}(2 u) \Sigma_{c d}^{j, m}(-u)+\delta_{a b} e(2 u) \\
& \cong \frac{u(u+i / 2)\left(u^{2}+(j+1 / 2)^{2}\right)}{\left(u^{2}+(m+1 / 2)^{2}\right)\left(u^{2}+(m-1 / 2)^{2}\right)}\left(\begin{array}{cc}
1 & \frac{m}{u+i / 2} \\
-\frac{m}{u+i / 2} & 1
\end{array}\right) \otimes\left(\begin{array}{cc}
1 & \frac{m}{u+i / 2} \\
-\frac{m}{u+i / 2} & 1
\end{array}\right) .
\end{aligned}
$$


Here we also showed how the $\mathfrak{s o}(4) K$-matrix can be factorized into two $\mathfrak{s u}(2)$ factors. Based on the $\mathfrak{s u}(2)$ overlaps our proposition for the full overlap is

$$
\begin{aligned}
& \frac{\left\langle\operatorname{MPS}_{k} \mid \mathbf{u}, \mathbf{v}, \mathbf{w}\right\rangle}{\sqrt{\langle\mathbf{u}, \mathbf{v}, \mathbf{w} \mid \mathbf{u}, \mathbf{v}, \mathbf{w}\rangle}}= \\
& \sum_{m=-j}^{j} m^{L} \prod_{i=1}^{N} \frac{u_{i}\left(u_{i}+\frac{i}{2}\right)\left(u_{i}^{2}+\frac{(2 j+1)^{2}}{4}\right)}{\left(u_{i}^{2}+\frac{(2 m-1)^{2}}{4}\right)\left(u_{i}^{2}+\frac{(2 m+1)^{2}}{4}\right)} \prod_{i=1}^{M_{+}} \frac{v_{i}^{2}+m^{2}}{v_{i}\left(v_{i}+\frac{i}{2}\right)} \prod_{i=1}^{M_{-}} \frac{w_{i}^{2}+m^{2}}{w_{i}\left(w_{i}+\frac{i}{2}\right)} \sqrt{\frac{\operatorname{det} G_{+}}{\operatorname{det} G_{-}}}= \\
& \sum_{m=-j}^{j} m^{L} \prod_{i=1}^{N} \frac{Q_{1}(i j+i / 2) Q_{+}(i m) Q_{-}(i m)}{Q_{1}(i m+i / 2) Q_{1}(i m-i / 2)} \sqrt{\frac{Q_{1}(0) Q_{1}(i / 2)}{Q_{+}(0) Q_{+}(i / 2) Q_{-}(0) Q_{-}(i / 2)} \frac{\operatorname{det} G_{+}}{\operatorname{det} G_{-}}}
\end{aligned}
$$

which again agrees with the numerically proved formula [13]. In (3.47) we used the $\mathfrak{s o}(6)$ $Q$-functions

$$
Q_{1}(u)=\prod_{i=1}^{N}\left(u-u_{i}\right), \quad Q_{+}(u)=\prod_{i=1}^{N}\left(u-v_{i}\right), \quad Q_{-}(u)=\prod_{i=1}^{N}\left(u-w_{i}\right) .
$$

\subsection{Proposal for the asymptotic overlap in AdS/dCFT for generic $\mathrm{k}$}

Comparing the $\mathfrak{s o}(6)$ result (3.46) with the weak coupling limit of section 2.4 it is natural to assume a diagonal K-matrix structure in the boundary space consisting of the diagonal elements

$$
\mathbb{K}^{A d S / d C F T}=\operatorname{diag}\left(\mathbb{K}^{(j, j)}(u), \mathbb{K}^{(j, j-1)}(u), \ldots, \mathbb{K}^{(j, 1-j)}(u), \mathbb{K}^{(j,-j)}(u)\right)
$$

where $\mathbb{K}^{(j, m)}(u)$ is defined in (2.28) and the parameters are fixed as $k_{1}=k_{4}=1$ and $k_{2}=0$. Thus the one-point function in the asymptotic limit can be calculated as the overlap of the boundary state $B_{k}$ based on this K-matrix and the periodic multi-particle state

$$
c_{\Delta}=\frac{\left\langle\mathrm{B}_{k} \mid \mathbf{u}, \mathbf{y}^{(\nu)}, \mathbf{w}^{(\nu)}\right\rangle}{\sqrt{\left\langle\mathbf{u}, \mathbf{y}^{(\nu)}, \mathbf{w}^{(\nu)} \mid \mathbf{u}, \mathbf{y}^{(\nu)}, \mathbf{w}^{(\nu)}\right\rangle}}=\sum_{m=-j}^{j} \frac{x_{m}^{L}\left\langle\mathrm{~B}^{(j, m)} \mid \mathbf{u}, \mathbf{y}^{(\nu)}, \mathbf{w}^{(\nu)}\right\rangle}{\sqrt{\left\langle\mathbf{u}, \mathbf{y}^{(\nu)}, \mathbf{w}^{(\nu)} \mid \mathbf{u}, \mathbf{y}^{(\nu)}, \mathbf{w}^{(\nu)}\right\rangle}}
$$

where the Bethe states are labeled by the Bethe roots $\mathbf{y}^{(\nu)}, \mathbf{w}^{(\nu)}$ with $\nu=1,2$ of the two $\mathfrak{s u}(2 \mid 2)$ wings. The matrix product type boundary state involves a trace coming from the boundary inner degree of freedom. Since the K-matrix is diagonal the overlap is simply the sum of individual overlaps with $\left\langle B^{(j, m)}\right|$, which is the boundary state built from the scalar (in boundary space) K-matrix $\mathbb{K}^{(j, m)}$. To decide how to normalize the individual components we used the results of [17], which was obtained from exact localization calculations and also from bootstrap considerations and was checked against perturbative calculations [10]. Fortunately we have already calculated the overlap (3.50) in [18] thus the 
full result we propose for the one point function is

$$
c_{\Delta}=\sum_{m=-j}^{j} x_{m}^{L} \prod_{i=1}^{N / 2} K_{j, m}\left(u_{i}\right) \prod_{\nu=1}^{2} \prod_{i=1}^{M_{1}^{(\nu)} / 2} \frac{\left(y_{i}^{(\nu)}\right)^{2}+x_{m}^{2}}{i x_{m} y_{i}^{(\nu)}} \prod_{i=1}^{\left\lfloor M_{1}^{(\nu)} / 4\right\rfloor} \frac{1}{w_{i}^{(\nu)}\left(w_{i}^{(\nu)}+\frac{i}{2 g}\right)} \sqrt{\frac{\operatorname{det} G_{+}}{\operatorname{det} G_{-}}}
$$

where the Gaudin like determinants can be calculated from the BA equations [18].

\section{Conclusions}

We formulated and analyzed the boundary state bootstrap for factorizing K-matrices with residual osp $(2 \mid 2)_{c}$ symmetry. This K-matrix had three "bosonic" and one "fermionic" orientations parameterized by $k_{i}$ and $x_{s}$, respectively. By bootstrapping on the bound state pole we showed that the matrix structure of the excited boundary state had the same form as the ground state one, merely the parameter $x_{s}$ was shifted as $x_{s \pm 1}$. We then analyzed the pole structure of the excited boundary K-matrix and identified a new boundary bound state. For semi integer $s$ we closed the boundary bootstrap by providing the full set of boundary bound states together with their K-matrices and explanations of all of their poles. In order to apply these results for higher dimensional MPS in the D3-D5 AdS/dCFT setting we calculated the tree level excitation K-matrix. We did it first in an $\mathfrak{s u}(3)$ spin chain, then we extended the result for the $\mathfrak{s o}(6)$ spin chain. In both cases the excitation K-matrices were diagonal in the boundary space without any inner degree of freedom. By generalizing our nesting method developed in [18] for matrix product states we calculated the most general overlaps at tree level. These agreed with previous results in the literature $[11,13]$. The comparison of the tree level excitation K-matrix with the weak coupling limit of our all loop K-matrix fixed its parameters. Based on our previous results we could propose the all loop asymptotic overlaps for the D3-D5 system including all excitations. These extend the results of [17] from the diagonal $\mathfrak{s u}(2)$ sector for the full theory.

Our results are asymptotic in the sense that it does not contain any wrapping corrections. To incorporate those effects of the virtual particles one has to go through the program suggested in $[17,32,33]$ The recent results [34-37] on excited state g-functions could help to incorporate the wrapping corrections also in our case, while the result [37] might sheds some light how it could be formulated more efficiently.

There are two ways to embed the residual $\mathfrak{o s p}(2 \mid 2)_{c}$ symmetry into $\mathfrak{s u}(2 \mid 2)_{c}$ depending on which $\mathfrak{s u}(2)$ factor is unbroken. Here we analyzed the case with the full Lorentzian symmetry. Completely analogous considerations can be performed for the case when the R-symmetry is unbroken. Those results can be relevant for correlation functions with Wilson loops.

\section{Acknowledgments}

We thank the NKFIH research Grants K116505 and K134946 for support. 
Open Access. This article is distributed under the terms of the Creative Commons Attribution License (CC-BY 4.0), which permits any use, distribution and reproduction in any medium, provided the original author(s) and source are credited.

\section{References}

[1] S. Ghoshal and A.B. Zamolodchikov, Boundary $S$ matrix and boundary state in two-dimensional integrable quantum field theory, Int. J. Mod. Phys. A 9 (1994) 3841 [Erratum ibid. 9 (1994) 4353] [hep-th/9306002] [INSPIRE].

[2] J.-S. Caux and F.H.L. Essler, Time evolution of local observables after quenching to an integrable model, Phys. Rev. Lett. 110 (2013) 257203 [arXiv:1301.3806] [INSPIRE].

[3] L. Piroli, E. Vernier, P. Calabrese and B. Pozsgay, Integrable quenches in nested spin chains I: the exact steady states, J. Stat. Mech. 1906 (2019) 063103 [arXiv:1811.00432] [INSPIRE].

[4] L. Piroli, E. Vernier, P. Calabrese and B. Pozsgay, Integrable quenches in nested spin chains II: fusion of boundary transfer matrices, J. Stat. Mech. 1906 (2019) 063104 [arXiv: 1812.05330] [INSPIRE].

[5] B. Pozsgay, L. Piroli and E. Vernier, Integrable Matrix Product States from boundary integrability, SciPost Phys. 6 (2019) 062 [arXiv: 1812.11094] [INSPIRE].

[6] M. de Leeuw, Coordinate Bethe Ansatz for the String S-matrix, J. Phys. A 40 (2007) 14413 [arXiv: 0705.2369] [INSPIRE].

[7] M. de Leeuw, C. Kristjansen and K. Zarembo, One-point Functions in Defect CFT and Integrability, JHEP 08 (2015) 098 [arXiv: 1506.06958] [INSPIRE].

[8] I. Buhl-Mortensen, M. de Leeuw, C. Kristjansen and K. Zarembo, One-point Functions in AdS/dCFT from Matrix Product States, JHEP 02 (2016) 052 [arXiv: 1512.02532] [INSPIRE].

[9] M. de Leeuw, C. Kristjansen and G. Linardopoulos, One-point functions of non-protected operators in the $\mathrm{SO}(5)$ symmetric D3-D7 dCFT, J. Phys. A 50 (2017) 254001 [arXiv: 1612.06236] [INSPIRE].

[10] I. Buhl-Mortensen, M. de Leeuw, A.C. Ipsen, C. Kristjansen and M. Wilhelm, One-loop one-point functions in gauge-gravity dualities with defects, Phys. Rev. Lett. 117 (2016) 231603 [arXiv: 1606.01886] [INSPIRE].

[11] M. de Leeuw, C. Kristjansen and S. Mori, AdS/dCFT one-point functions of the SU(3) sector, Phys. Lett. B 763 (2016) 197 [arXiv:1607.03123] [inSPIRE].

[12] I. Buhl-Mortensen, M. de Leeuw, A.C. Ipsen, C. Kristjansen and M. Wilhelm, Asymptotic One-Point Functions in Gauge-String Duality with Defects, Phys. Rev. Lett. 119 (2017) 261604 [arXiv: 1704.07386] [INSPIRE].

[13] M. De Leeuw, C. Kristjansen and G. Linardopoulos, Scalar one-point functions and matrix product states of AdS/dCFT, Phys. Lett. B $\mathbf{7 8 1}$ (2018) 238 [arXiv:1802.01598] [InSPIRE].

[14] M. De Leeuw, T. Gombor, C. Kristjansen, G. Linardopoulos and B. Pozsgay, Spin Chain Overlaps and the Twisted Yangian, JHEP 01 (2020) 176 [arXiv: 1912.09338] [INSPIRE].

[15] G. Linardopoulos, Solving holographic defects, PoS (CORFU2019) 141 [arXiv: 2005.02117] [INSPIRE].

[16] C. Kristjansen, D. Müller and K. Zarembo, Integrable boundary states in D3-D5 dCFT: beyond scalars, JHEP 08 (2020) 103 [arXiv: 2005.01392] [INSPIRE].

[17] S. Komatsu and Y. Wang, Non-perturbative defect one-point functions in planar $\mathcal{N}=4$ super-Yang-Mills, Nucl. Phys. B 958 (2020) 115120 [arXiv:2004.09514] [INSPIRE]. 
[18] T. Gombor and Z. Bajnok, Boundary states, overlaps, nesting and bootstrapping AdS/dCFT, JHEP 10 (2020) 123 [arXiv: 2004.11329] [INSPIRE].

[19] P. Dorey, R. Tateo and G. Watts, Generalizations of the Coleman-Thun mechanism and boundary reflection factors, Phys. Lett. B 448 (1999) 249 [hep-th/9810098] [INSPIRE].

[20] P. Mattsson and P. Dorey, Boundary spectrum in the sine-Gordon model with Dirichlet boundary conditions, J. Phys. A 33 (2000) 9065 [hep-th/0008071] [INSPIRE].

[21] Z. Bajnok, L. Palla and G. Takács, Boundary states and finite size effects in sine-Gordon model with Neumann boundary condition, Nucl. Phys. B 614 (2001) 405 [hep-th/0106069] [INSPIRE].

[22] Z. Bajnok, L. Palla, G. Takács and G.Z. Toth, The Spectrum of boundary states in sine-Gordon model with integrable boundary conditions, Nucl. Phys. B 622 (2002) 548 [hep-th/0106070] [INSPIRE].

[23] Z. Bajnok, G. Bohm and G. Takács, On perturbative quantum field theory with boundary, Nucl. Phys. B 682 (2004) 585 [hep-th/0309119] [INSPIRE].

[24] N. Beisert et al., Review of AdS/CFT Integrability: An Overview, Lett. Math. Phys. 99 (2012) 3 [arXiv: 1012.3982] [INSPIRE].

[25] G. Arutyunov and S. Frolov, Foundations of the $A d S_{5} \times S^{5}$ Superstring. Part I, J. Phys. A 42 (2009) 254003 [arXiv:0901.4937] [INSPIRE].

[26] G. Arutyunov and S. Frolov, On String S-matrix, Bound States and TBA, JHEP 12 (2007) 024 [arXiv: 0710.1568] [INSPIRE].

[27] L. Piroli, B. Pozsgay and E. Vernier, What is an integrable quench?, Nucl. Phys. B 925 (2017) 362 [arXiv:1709.04796] [INSPIRE].

[28] M. Brockmann, J. De Nardis, B. Wouters and J.-S. Caux, A Gaudin-like determinant for overlaps of Néel and XXZ Bethe states, J. Phys. A 47 (2014) 145003.

[29] M. Brockmann, J. De Nardis, B. Wouters and J.-S. Caux, Néel-XXZ state overlaps: odd particle numbers and Lieb-Liniger scaling limit, J. Phys. A 47 (2014) 345003.

[30] B. Pozsgay, Overlaps with arbitrary two-site states in the XXZ spin chain, J. Stat. Mech. 1805 (2018) 053103 [arXiv: 1801.03838] [INSPIRE].

[31] Y. Jiang and B. Pozsgay, On exact overlaps in integrable spin chains, JHEP 06 (2020) 022 [arXiv: 2002.12065] [INSPIRE].

[32] Y. Jiang, S. Komatsu and E. Vescovi, Structure constants in $\mathcal{N}=4$ SYM at finite coupling as worldsheet g-function, JHEP 07 (2020) 037 [arXiv: 1906.07733] [INSPIRE].

[33] Y. Jiang, S. Komatsu and E. Vescovi, Exact Three-Point Functions of Determinant Operators in Planar $N=4$ Supersymmetric Yang-Mills Theory, Phys. Rev. Lett. 123 (2019) 191601 [arXiv: 1907.11242] [INSPIRE].

[34] I. Kostov, D. Serban and D.-L. Vu, Boundary TBA, trees and loops, Nucl. Phys. B 949 (2019) 114817 [arXiv:1809.05705] [INSPIRE].

[35] D.-L. Vu, I. Kostov and D. Serban, Boundary entropy of integrable perturbed $\mathrm{SU}(2)_{k} W Z N W$, JHEP 08 (2019) 154 [arXiv: 1906.01909] [INSPIRE].

[36] I. Kostov, Effective Quantum Field Theory for the Thermodynamical Bethe Ansatz, JHEP 02 (2020) 043 [arXiv: 1911.07343] [INSPIRE].

[37] J. Caetano and S. Komatsu, Functional equations and separation of variables for exact g-function, JHEP 09 (2020) 180 [arXiv:2004.05071] [INSPIRE]. 